\title{
Traduire
}

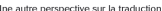

Revue française de la traduction

$229 \mid 2013$

L'environnement, une spécialisation durable?

\section{Le développement durable et le monde des grandes entreprises}

\section{Ginny Hill}

Traducteur : Géraldine Chantegrel

\section{OpenEdition \\ Journals}

Édition électronique

URL : http://journals.openedition.org/traduire/587

DOI : $10.4000 /$ traduire.587

ISSN : 2272-9992

Éditeur

Société française des traducteurs

Édition imprimée

Date de publication : 15 décembre 2013

Pagination : 41-48

ISSN : 0395-773X

Référence électronique

Ginny Hill, «Le développement durable et le monde des grandes entreprises », Traduire [En ligne], 229 |

2013, mis en ligne le 15 décembre 2015, consulté le 10 décembre 2020. URL : http://

journals.openedition.org/traduire/587 ; DOI : https://doi.org/10.4000/traduire.587 


\section{Le développement durable et le monde des grandes entreprises}

\section{Ginny Hill}

Le concept du développement durable a eu des répercussions manifestes sur les technologies et les prestations commercialisées par les grandes entreprises. Son impact sur les stratégies et les méthodes de travail, bien que moins évident, ne cesse de croître.

Depuis 2001, les sociétés cotées à la Bourse de Paris ont l'obligation d'intégrer des données sociales et environnementales dans leurs rapports annuels. Beaucoup de grands groupes ont choisi de publier un document distinct, donnant ainsi naissance au " rapport développement durable ". En 2007, le CODEVI (COmpte pour le DEVeloppement Industriel) a cédé la place au Livret de développement durable, un livret d'épargne défiscalisé principalement créé pour collecter des fonds destinés à financer des travaux d'économie d'énergie dans les bâtiments anciens.

Est-ce depuis cette époque que le " développement durable " est sur toutes les lèvres ? En France, les bulletins d'information et les sites internet des collectivités locales en regorgent. Que ce soit dans le domaine des transports, des performances énergétiques ou de l'aide à l'emploi, nombreuses sont désormais les activités qui se mesurent à l'aune du développement durable.

Pourtant, ce concept a mis du temps à passer dans le langage courant. La définition que l'on cite le plus souvent est tirée du Rapport " Brundtland ", " Notre avenir à tous(1) " : "Le développement durable est un développement qui répond aux besoins du présent sans compromettre la capacité des générations futures de répondre aux leurs. " Ce rapport date de 1987.

\section{Considérations linguistiques}

Lorsque l'expression " développement durable " a commencé à apparaître régulièrement dans les publications d'entreprise (vers la fin des années 1990 ?), elle était souvent traduite à tort

(1) Cf. http://www.diplomatie.gouv.fr/fr/sites/odyssee-developpement-durable/files/5/rapport_brundtland.pdf 
en langue anglaise par durable development. Aujourd'hui, une telle erreur, qui trahissait une certaine méconnaissance du sujet, me semble peu probable.

En ce qui concerne le terme en lui-même, le débat autour du mot " développement " reste enflammé. En effet, "développement " signifie " croissance ", ce qui soulève la question suivante : l'avenir de la planète doit-il encore s'envisager sous l'angle de la croissance, ou plutôt sous celui de la " décroissance " ? Si le terme "durabilité " (sustainability en anglais) est plus neutre, je dois avouer qu'en tant que traductrice œuvrant pour le secteur privé, aucun de mes clients n'est prêt à renoncer à la croissance(2)...

En Europe, les trois piliers du développement durable sont habituellement désignés sous le triptyque de "performance économique, environnementale et sociale ". Cela dit, beaucoup de mes clients français ont tendance à scinder la " performance sociale " en données "sociales " et " sociétales ". Les premières concernent les ressources humaines, tandis que les secondes font souvent référence au mécénat d'entreprise, par exemple les actions réalisées au niveau des collectivités ou l'aide internationale. Différents termes comme outreach (" actions de proximité ") ou work in the community (" travaux en prise directe avec les collectivités locales ") sont utilisés selon le contexte et les activités menées. Par contre, societal performance est un calque que l'on trouve rarement dans les publications en langue anglaise.

Mentionnons également le concept de "triple bilan " (triple bottom line), promu par les Nations Unies et le Conseil international pour les initiatives écologiques locales (ICLEI). Le triple bilan introduit l'idée que le résultat des entreprises doit intégrer les aspects sociaux, environnementaux et économiques ou, en termes plus parlants, les profits, les personnes et la planète (les trois P). Cette approche est plutôt l'apanage du secteur public, la " responsabilité sociale des entreprises " étant son équivalent dans le secteur privé(3).

\section{Évolution de l'attitude des entreprises}

Les industries liées à l'environnement (en particulier les secteurs de l'eau, des déchets et de l'énergie) sont depuis une vingtaine d'années mon principal domaine d'activité. Ce sont des sujets qui touchent notre vie quotidienne et il est frappant de constater l'influence qu'a pu exercer petit à petit le concept de développement durable sur la mentalité des grandes entreprises. Initialement plutôt méfiantes, elles avaient tendance à considérer le développement

(2) The Hidden Messages Within Sustainable Development, écrit en 1994 par l'ingénieure et universitaire australienne Sharon Beder, offre un bon aperçu de la complexité du débat. (http://www.uow.edu.au/ sharonb/esd/ alternatives.html)

(3) Pour en savoir plus, voir http://en.wikipedia.org/wiki/Triple_bottom_line. 
durable comme une nouvelle source de contraintes et de règlements génératrice de coûts supplémentaires (aux États-Unis, où le concept a plus de mal à passer dans les mœurs qu'en Europe, je crains fort que de nombreux secteurs n'aient pas encore dépassé ce stade).

En France, face à la nécessité d'élaborer des rapports sur le développement durable, beaucoup d'entreprises ont d'abord pensé : "Du travail et des dépenses supplémentaires. Quelle perte de temps! ". Mais au fil des années, elles ont finalement adhéré à cette notion et l'ont intégrée dans leur stratégie. Si les industries liées à l'environnement ont bien évidemment été parmi les premières à adopter une telle démarche, elles sont loin d'être les seules. Certains de mes clients évoluant dans des domaines aussi variés que la construction, l'agroalimentaire et le commerce de détail partagent ces valeurs.

Le dénominateur commun est que toutes ces entreprises sont conscientes des gisements d'efficacité que recèle une telle démarche et de l'impact positif que peuvent avoir leurs politiques environnementales et sociales sur leur image, en leur offrant d'excellents sujets de communication et en attirant des actionnaires socialement responsables.

\section{Des procédés et des outils nouveaux}

Les changements apportés par le développement durable sont pourtant tout sauf superficiels. Pour produire les données qu'elles ont l'obligation de publier, les sociétés françaises ont dû mettre en place des systèmes performants. Recueillir des données cohérentes auprès de centaines de filiales, dont beaucoup se trouvent à l'étranger et sont donc soumises à des législations nationales différentes, n'est pas une mince affaire. Nombre de grandes entreprises continuent d'affiner et d'améliorer leurs systèmes de rapports tout en élargissant le périmètre de leurs chiffres.

Au lieu de se contenter de communiquer des données chiffrées, certaines entreprises ont choisi de profiter de l'occasion pour jeter des bases leur permettant de fixer des objectifs à plus long terme dans le cadre d'une stratégie active d'amélioration de leur performance environnementale et sociale. Les objectifs environnementaux ainsi définis visent généralement la réduction des gaz à effet de serre, la limitation de la consommation d'eau et d'énergie et la protection de la biodiversité. Les objectifs de performance sociale, quant à eux, sont axés sur la santé et la sécurité (notamment la prévention des accidents), l'égalité des sexes et la diversité. Pour appliquer leur nouvelle stratégie, les entreprises ont dû mettre en place des indicateurs fiables destinés à servir de base à leurs objectifs, puis mesurer leurs performances par rapport à ces objectifs. Les organisations en question, souvent de très grande taille, ont créé des systèmes de gestion environnementale ainsi que d'autres outils à des fins de cohérence et en vue d'aider leurs cadres du monde entier dans la mise en œuvre de la stratégie. Chartes de développement durable et codes d'éthique fixent le cadre général et constituent une 
vitrine permettant de présenter ces politiques au monde extérieur. Des questionnaires d'autodiagnostic ont été élaborés en interne, parfois basés sur des principes comme ceux du Pacte mondial des Nations Unies, des conventions de l'Organisation internationale du Travail et de la norme de responsabilité sociale ISO 26000. Aujourd'hui, beaucoup d'entreprises insistent également pour que leurs principaux fournisseurs se conforment à leurs politiques sociales et environnementales, élargissant ainsi leur périmètre d'influence selon les exigences du Pacte mondial. Certaines procèdent à la formation de leurs fournisseurs pour aider ces derniers à relever leur niveau de performance.

Les différents processus de certification sont, pour leur part, autant d'outils capables de pousser les entreprises vers des améliorations de leur performance sociale et environnementale. Citons notamment l'ISO 14000 pour la gestion environnementale, I'ISO 9000 pour la gestion de la qualité et l'OHSAS 18000 pour la santé et la sécurité au travail. Les communiqués de presse annonçant triomphalement une double ou une triple certification montrent l'importance que l'on accorde à ces labels devenus pour beaucoup une garantie de sérieux et de maturité. Au final, le principal avantage de telles certifications est peut-être celui d'avoir franchi les étapes nécessaires à leur obtention. Grâce à ces normes, les entreprises disposent de bases solides pour procéder à des réflexions approfondies sur la manière de travailler et pour instaurer de nouvelles méthodes inspirées des meilleures pratiques internationales.

Le Pacte mondial des Nations Unies(4), lancé en 2000, constitue un autre vecteur de progrès. Les entreprises participantes s'engagent à appliquer les dix principes du Pacte, qui sont axés sur les droits de l'homme, les conditions de travail, l'environnement et la lutte contre la corruption. Elles doivent également s'engager à faire état chaque année des progrès réalisés dans l'application de ces principes, ce qui assure une amélioration continue et favorise une plus grande notoriété du Pacte mondial. L'absence de telles informations pendant deux ans d'affilée entraîne automatiquement l'exclusion de l'entreprise défaillante. À ce jour, 970 sociétés françaises ont souscrit au Pacte ; seules cinq entreprises du CAC 40 manquent à l'appel.

L'inscription à des indices de développement durable comme le DISI (indice de développement durable du Dow Jones) et l'ASPI Eurozone constitue un autre défi de taille. En effet, ces indices sont suivis de près par les investisseurs désireux de privilégier les entreprises connues pour leur respect de l'environnement et pour leurs comportements éthiques. La concurrence est rude pour intégrer ces indices. Ils sont actualisés chaque année et leurs critères de sélection évoluent régulièrement. Pour continuer de figurer sur la liste, les entreprises doivent améliorer constamment leur performance économique, environnementale et sociale.

(4) http://www.pactemondial.org/ 


\section{Le développement durable, un atout concurrentiel}

En France, de plus en plus de marchés publics contiennent désormais une clause obligeant les attributaires à réserver un certain nombre d'emplois à des personnes bénéficiant de programmes d'insertion professionnelle, ou plus généralement aux habitants de la région. Un grand projet d'infrastructure, par exemple, peut avoir d'importantes répercussions sur l'emploi local et permettre à des personnes d'avoir accès à une formation et d'acquérir une expérience professionnelle, facilitant ainsi leur insertion dans le monde du travail. Le maître d'œuvre de la LGV SEA Tours-Bordeaux, en cours de construction, s'est engagé à recruter et former 1200 personnes sélectionnées dans les environs, dont 400 dans le cadre de programmes d'insertion professionnelle. Pôle Emploi a mis en place une unité de coordination régionale afin de travailler avec les ressources humaines de ce maître d'œuvre et d'assurer la réussite du processus de recrutement. Pour aider ses petites filiales à respecter ces clauses en matière d'emploi, l'entreprise a même créé une filiale spécialement chargée de conseiller et d'épauler les équipes RH. Loin de la générosité forcée, elle n’a jamais caché le fait que son objectif était de transformer une contrainte en valeur ajoutée. L'existence d'une telle filiale est un plus pour les appels d'offres et constitue un avantage concurrentiel.

D'une manière générale, cette tendance à adopter une approche résolument pragmatique est l'une des évolutions auxquelles j'ai assisté. Elle est motivée, me semble-t-il, par un souci de transparence (et donc de bonne gouvernance), mais aussi par le souhait de montrer aux investisseurs que la rentabilité reste une priorité et que l'argent consacré à ces initiatives est bien investi. Le civisme angélique d'hier a cédé la place à la franchise : "Si nous agissons ainsi, c'est parce qu'il y va de notre intérêt commercial».

Le message en filigrane est que les sociétés du secteur privé revendiquent le droit et le besoin de réaliser des bénéfices. Condamnés par certaines ONG et organisations à but non lucratif qui diabolisent les entreprises commerciales et estiment qu'elles sont juste bonnes à dégrader l'environnement et à exploiter le travailleur moyen, les grands groupes contre-attaquent en faisant valoir le rôle majeur qu'ils jouent dans l'économie, les emplois qu'ils créent et la principale ressource que constitue leur personnel, raison pour laquelle ils en prennent soin. Dans les communications d'entreprise, l'on insiste constamment sur les montants importants investis en $R \& D$ pour produire les technologies et les services capables d'assurer le développement durable auquel tout le monde aspire.

\section{Dialoguer avec les parties prenantes}

L'instauration d'un " dialogue avec les parties prenantes " est peut-être la démarche la plus récente. S'il s'agit en fait d'une évolution très progressive, aujourd'hui, certaines entreprises ont pleinement adopté ce principe et y voient un levier essentiel pour la conception et l'élaboration de grands projets. 
Leurs motivations sont relativement simples et s'inscrivent dans la droite ligne de leur mantra : "Si nous agissons ainsi, c'est parce qu'il y va de notre intérêt commercial ". Le but est d'assurer l'" acceptabilité " d'un projet et de veiller à son bon déroulement.

Les grands projets d'infrastructure se heurtent souvent à des résistances. Selon l'Oxford English Dictionary, l'acronyme NIMBY (Not In My Backyard, c'est-à-dire "Pas dans mon jardin ") serait apparu en 1980, mais le concept de résistance locale organisée face à un projet jugé importun daterait plutôt des années 1950.

Auparavant, les entreprises avaient tendance à dire : "Nous ne faisons que construire, l'implantation du projet n'a rien à voir avec nous. Si vous n'êtes pas contents, ce n'est pas notre problème. " Mais les marchés du type " construction-exploitation-transfert " ont vu le jour et les grandes sociétés ont commencé à financer une grande partie, voire l'intégralité de certains projets en tablant sur le contrat d'exploitation pour assurer la rentabilité de l'affaire. Si jamais ce contrat était résilié face à l'opposition tenace d'un public résolument réfractaire au projet, l'attributaire s'exposait à d'importantes pertes financières et, de surcroît, à une publicité dommageable pour son image.

Un autre problème intervenant plus en amont était celui des protestations qui pouvaient retarder les projets pendant des mois, voire des années. Dans de tels cas, le maître d'œuvre se trouvait dans l'obligation de consacrer du temps, de l'argent et beaucoup de nuits blanches à tenter de convaincre les réfractaires de l'utilité et de la nécessité du projet.

On a donc assisté à un processus d'apprentissage en plusieurs étapes :

- nous ne participerons pas à des appels d'offres tant que nous n'aurons pas la certitude que la population pourra payer l'intégralité du prix du service proposé ;

- on ne doit pas attendre que la population paie la totalité du service proposé ;

- nous ne participerons à des appels d'offres que si nous avons la certitude que la population acceptera le projet et pourra payer le prix prévu ;

- nous devons intervenir en amont pour aider le maître d'ouvrage à faire accepter le projet par la population ;

- nous devons tenter de savoir quels sont les arguments contre le projet afin d'y trouver la parade ;

- nous devons tenter de savoir quels sont les arguments contre le projet afin de les prendre en compte et d'élaborer des solutions adaptées en concertation avec les opposants.

Aujourd'hui, il s'agit de travailler aussi en amont que possible avec le maître d'ouvrage dans le but de présenter le projet à toutes les parties prenantes, de tenir compte de leurs préoccupations dès la phase de conception et, avec un peu de chance, de pouvoir ainsi entreprendre les phases de construction et d'exploitation sans trop craindre de difficultés. 
Mais qui sont en fait les parties prenantes ? Pour les identifier, définir leurs exigences et décider de la manière d'y faire face, il faut mettre au point des méthodes et des outils.

Cette démarche, lorsqu'elle est réussie, se traduit par des projets qui tiennent compte des préoccupations des intéressés et assure des phases de construction et d'exploitation sans heurt. Au final, il s'agit d'une approche " gagnant-gagnant " qui a des répercussions positives sur les trois aspects de la performance d'une entreprise (économique, environnementale et sociale) et permet aux associations à but non lucratif de faire valoir leurs compétences. Au lieu de se considérer comme des ennemis à abattre, il importe que chacun respecte le point de vue de l'autre et que tout le monde travaille en bonne intelligence pour parvenir à la meilleure solution.

\section{Révolutions stratégiques}

La nécessité de respecter différentes obligations règlementaires, de tenir compte des besoins des populations locales et de bien comprendre les avantages pouvant découler de ces contraintes a donné naissance à un mélange de mesures d'encouragement et de mesures coercitives qui ont eu un impact concret sur les méthodes de travail et les stratégies de communication des entreprises.

Cela dit, les principes du développement durable ont des effets bien plus profonds sur les entreprises, qui pourraient à eux seuls faire l'objet d'un autre article. Prenons par exemple le secteur de l'eau. II y a peu de temps encore, les revenus de ces entreprises étaient tributaires des volumes d'eau vendus. Pour elles, la configuration idéale consistait en un hiver pluvieux pour reconstituer les réserves, suivi d'un été chaud et sec où tout le monde jouerait du tuyau pour arroser son jardin ou sa pelouse. Aujourd'hui, les sociétés de distribution d'eau dépensent des sommes importantes pour suggérer des astuces à leurs clients et leur expliquer comment réduire leur consommation. Ces entreprises sont, en effet, prises dans un cercle vicieux : les sources d'eau étant de plus en plus polluées, il faut des traitements de plus en plus poussés pour les rendre potables, d'où une augmentation des coûts de traitement ; en même temps, elles doivent investir dans des mesures de réduction de la consommation et subissent une énorme pression de la part des municipalités qui exigent des baisses tarifaires. Elles doivent donc rechercher de nouvelles sources de revenus et élaborer des plans stratégiques novateurs. D'où l'idée révolutionnaire d'indexer leur rémunération sur les économies d'eau réalisées plutôt que sur les volumes écoulés.

L'influence croissante du développement durable ne devrait pas fléchir. En 2012, lors de la Conférence des Nations Unies sur le développement durable (connue sous le nom de Rio +20 ), il a été décidé " de lancer un processus visant à développer un ensemble d'objectifs de développement durable qui s'appuieront sur les objectifs du Millénaire pour le développement et 
convergeront avec le programme de développement pour l'après-2015(5) ". Le document final de Rio + 20 s'intitule "L'avenir que nous voulons ". Bien évidemment, nous aspirons tous à un monde qui ne connaîtrait ni la pauvreté ni la pollution ni la discrimination. D'ailleurs, je pense très sincèrement que les grandes entreprises ont un rôle important à jouer à cet égard. D'après mon expérience personnelle, d'énormes progrès ont déjà été réalisés dans ce sens et devraient se poursuivre si l'on sait manier habilement aussi bien le bâton que la carotte. Les plus cyniques diraient que le monde des affaires manifeste un intérêt de pure forme pour le développement durable, et ce dans le seul souci de respecter la législation nationale. Quand bien même, qui pourrait nier les avantages d'une amélioration des conditions de travail, d'une meilleure protection de l'environnement et d'une utilisation plus rationnelle des ressources de notre planète? Pourvu que cela dure...

ginny.hill@wanadoo.fr

\section{Traduit de l'anglais par Géraldine Chantegrel}

Ginny Hill est traductrice et réviseuse dans la paire linguistique français-anglais, et travaille également en tant que secrétaire de rédaction sur des supports en langue anglaise. Elle exerce son métier en France pour le compte de clients du monde commercial. Depuis le début des années 1990, elle évolue essentiellement dans les domaines de l'environnement (eau, gestion des déchets, énergie), du transport et du bâtiment, ce qui lui a valu le privilège de travailler à de nombreux projets liés au développement durable, à la mobilité et au développement urbain pour de grandes sociétés françaises.

(5) http://sustainabledevelopment.un.org/index.php?menu=1300 